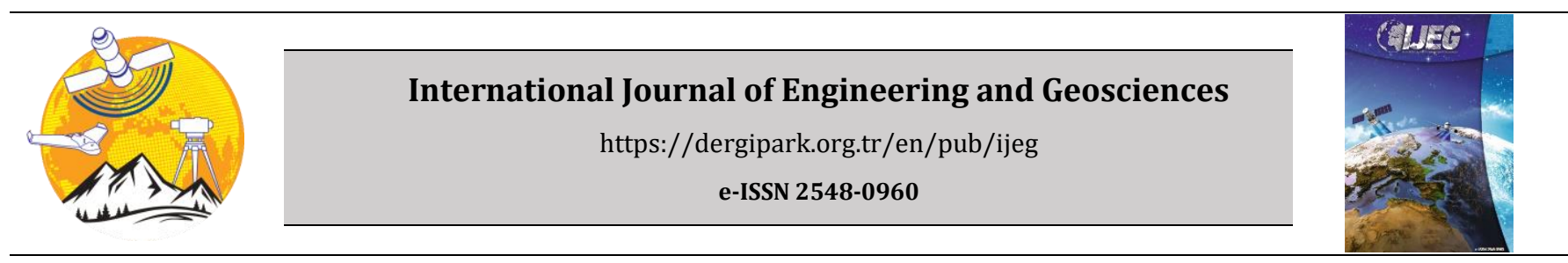

\title{
A fuzzy multi-criteria decision-making approach for the assessment of forest health applying hyper spectral imageries: A case study from Ramsar forest, North of Iran
}

\author{
Khalil Valizadeh Kamran1ㅁ, Behnam Khorrami² ${ }^{*}$ \\ ${ }^{1}$ University of Tabriz, Faculty of Planning and Environmental Sciences, Department of Remote Sensing and GIS, Tabriz, Iran \\ ${ }^{2}$ Dokuz Eylul University, Graduate School of Natural and Applied Sciences, Department of GIS, Izmir, Turkey
}

\author{
Keywords \\ Hyperspectral \\ Fuzzy set \\ Forest health \\ Vegetation indices \\ Ramsar
}

\begin{abstract}
The hyperspectral images have so far been widely utilized in monitoring and detecting the changes in a broad range of environmentally related matters. The hyperspectral image analysis yields maps that show spatial dispersion of physical and ecological characteristics of the terrain. Within the scope of the current study, an integrated Fuzzy-MCDM in a Geographic Information Systems (GIS) platform was used to map the health condition of Ramsar forest. Spectral indices can provide different methods for identifying vegetation coverings. For forest health analysis, spectral indices such as NDWI, CRI1, PSRI, PRI, and NDVI were used to infer the causative factors of forest health. The findings highlight the suitability of the used methodology in identifying potential forest statuses, where forest health protection measures can be taken in advance. The results also suggest that the southern and the western aspects of the study area are of "very low" to "low" forest health. Furthermore, the results indicate a high potentiality for applying the spatial MCDM techniques as an effective tool for the forest health investigation.
\end{abstract}

\section{INTRODUCTION}

The field-based assessment of forest health practices comprises the majority of the recent literature. The traditional field-based analysis is quite useful for investigating health conditions, however, the physiological variations which characterize the plants' early stress levels are not revealed through these approaches (Sampson et al., 2000). Assessment of the physiological conditions provides an indication of the productivity and resiliency to environmental pressures (Chapin, 1991; Colombo and Parker,1999) and can provide an early warning sign of a decline in stand vigor and productivity (Zarco et al. 2002). Hyperspectral images have been extensively tested in the areas of natural resources, plants, and surface water (Moroni et al. 2013). The existence of pigments (primarily chlorophyll-a, chlorophyll-b, xanthophylls, and carotenoids), as well as the canopy water portion, whose content differs according to the extent of the chemical as well as biological characteristics and the physical structure of plants, influence the spectral signature of vegetation (Sims and Gamon, 2002). The reflectance spectrum of plants may depict a variety of characteristics, including the severity of senescence, the diminishment of leaf structure, and any diseases or defects that may affect plants (Carter and Miller, 1994). By using spectral indices, Gamon and Surfus (1999) estimated the amount of chemical content in plants' leaves and canopies. These spectral indicators can be used as an inference tool for several significant ecophysiological characteristics taking into account the existing associations among the chemical content, leaf structure, and function. Thenkabail et al (2002) identified the best hyperspectral narrow bands for describing the characteristics of the crops in the visible and near-infrared (NIR) regions of the electromagnetic spectrum. They utilized the spectral indices of the vegetation coverages, calculated using the narrow and wide wavebands, to determine relationships with biophysical variables and crop yield.

Multi-Criteria Decision Making (MCDM) techniques have been developed to find out solutions for the problems arising from competing preferences among various criteria (Keeney et al. 1993). MCDM comes with 
a range of methods for organizing decision challenges, as well as for planning, assessing, and prioritizing future outcomes (Feizizadeh et al. 2012; Feizizadeh and Blaschke, 2012). Integrating the objective as well as subjective criteria into decision-making processes is needed for rational decision-making (Pomerol and Barba-Romero2000). MCDM approaches can be especially beneficial in a collaborative participatory setting, where they can provide a useful mechanism for addressing a variety of environmental issues, such as quantifying "fire risk" and "forest health."etc. (Saaty 1968; Malczewski 2002; Sadiq and Husain 2005).

Forest health mapping aids in evaluating forest diseases such as forest pests and blight, as well as determining where timber harvesting can take place. The current research aims to propose a forest health map for the study area by incorporating five different spectral indices with a Fuzzy package.

\section{METHODOLOGY}

\subsection{Study Area}

Ramsar forest is located within the geographic coordinates of $50^{\circ} 22^{\prime} 06^{\prime \prime}-50^{\circ} 59^{\prime} 31^{\prime \prime} \mathrm{N}$ and $36^{\circ} 11^{\prime} 08^{\prime \prime}-$ $36^{\circ} 45^{\prime} 06^{\prime \prime}$ E. It constitutes the majority of Ramsar city, the capital city of Mazandaran province, north of Iran (Figure 1). Mazandaran forests are among the main green hotspots of the country regarding the volume of the produced trees and other merits ascribed to them. These forests are graded from 1 to 3 with an area of 42,894 ha, 32,758 ha, and 52,972 ha respectively. The commercial and non-commercial utilization is $21,202 \mathrm{~m}^{3}$ and $32,173 \mathrm{~m}^{3}$ respectively. The climate of Ramsar is hot and humid in summer and mild in winter with an approximate Total Annual Precipitation (TAP) of 1200 $\mathrm{mm}$.

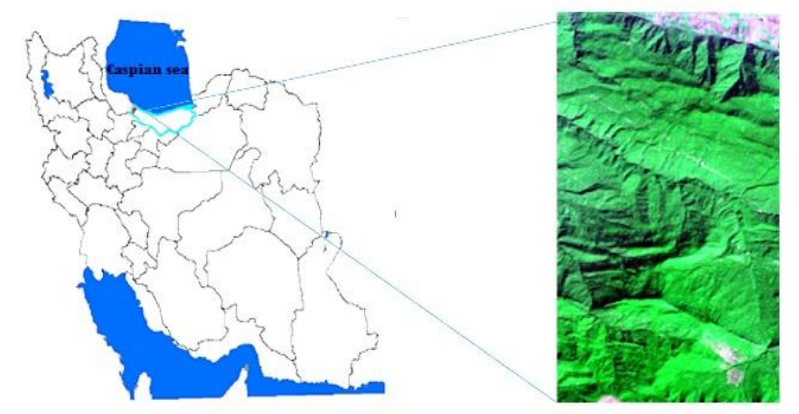

Figure 1. The geographic location of Ramsar Forest in North of Iran

\subsection{Methods}

The methodology applied for the current study is composed of three main components: (1), A Fuzzy Inference System for forest health (2), Fuzzy Degree Membership Functions, (3) Performing Scenarios. Different indices were measured, standardized, and combined in the fuzzy set with a suitable membership function to produce the final forest health map of the study area.

\subsubsection{Fuzzy Inference System for Forest Health}

In a multicriteria overlay analysis, the examination of the likelihood of the events representing a variety of sets is implemented through the Fuzzy overlay operations. Modeling human intelligence and real-world ideas can be assisted by fuzzy logic (FL) and fuzzy sets (FS) (Leondes, 1998). A fuzzy inference system (also called fuzzy model) is a nonlinear mapping process that encourages fuzzy inference and a series of "if-then-then" rules to derive its results.

Taking into account the shortcomings of the evaluation method, in particular, the complexity and limitations of the evaluation indicators, a comprehensive model of the health of forest ecosystems has been developed. A model can be obtained by combining the coefficient of variation method and fuzzy mathematical theory (Yan and Yang, 2011). Lotfi A. Zadeh proposed fuzzy logic as an intelligent control technology in 1968. This enables users to create models that reflect expert expertise and available measurements using a set of simple rules (Zadeh, 1968; Cox, 2005).

In fuzzy techniques, human knowledge is incorporated within the decision-making process through fuzzy mathematical algorithms. To date, several intelligent systems have been applied around the world to investigate the health status of forests annually (Yan and Yang, 2001) and to assess the risk posed by natural disasters (Iliadis, 2007). The fuzzy algebra claims that every phenomenon in the world is of an arbitrary fuzzy set (FS), and its degree of membership has a true value from 0 to 1 (Ilidias et al., 2007). Within the scope of this study, the spectral indices of NDWI, CRI1, PSRI, PRI, and NDVI were utilized to assess the health status of the forests in the surveyed area. A detailed description of the causal link to forest health is given in the following sections.

\subsubsection{Canopy Water Content}

Water is important for many plant processes, especially photosynthesis. The water characteristics, centered at $970 \mathrm{~nm}$ and $1190 \mathrm{~nm}$, can be easily measured on a hyperspectral sensor. The sampling of these spectral windows is usually not practical by multispectral sensors. Moisture content is an important number of plants to show healthier plants that are more likely to grow faster and become more refractory with higher moisture content (Peñuelas et al, 1992).

The water stress of plants is affected by both natural and agronomic plants (Boyer, 1982). Therefore, making use of spectral reflectance measurements for the precise investigation of the symptoms of water stress in plants is a central issue in remote sensing-oriented studies (Jackson, 1986). In forested areas, the dry season coincides with maximum insolation and temperature, causing special problems for the yearly growth of vegetation, which is severely curbed by summer water availability (Bolle et al., 2006). Water stress is manifested by plants due to reduced leaf area, stomatal conductivity, reduced $\mathrm{CO}_{2}$ absorption and associated photosynthesis, root elevation, and decreased development rate (Verstraeten et al., 2006). Total primary production 
(GPP), which represents global ecosystem photosynthesis, is a critical metric for identifying the most central ecosystem activities .

The water quantity among different plant species differs on account of the dissimilarities of leaf geometry, canopy structure, and water requirements. Even within a type of plant, considerable differences are depending on leaf thickness, water supply, and plant health. Moisture content was assessed using the NDWI (Normalized Difference Water Index).

\subsubsection{NDWI (Normalized Difference Water Index)}

The Normalized Difference Water Index (NDWI) $($ Gao, 1996) is one of the well-known spectral indices that measure the water content of plants using the spectral reflectance in the near-infrared (NIR) and short-wave infrared (SWIR) windows. SWIR reflectance band reflects changes in both the plant's moisture content as well as the spongy leaf structure of the plant canopy, whereas NIR reflectance describes the internal structure of the leaves as well as the leaves' dry content, but not the moisture content. The combined use of NIR and SWIR bands improves the accuracy of the water content detection from which the variations due to the inner structure and the dry content of the leaves, are removed (Ceccato et al. 2001).

NDWI has the same reflectivity at $857 \mathrm{~nm}$ and $1241 \mathrm{~nm}$, but it is susceptible to the variations in the moisture content of the plant canopy because of the slightly different absorption properties of the liquid water. Light scattering by the plant canopy improves the absorption of weak liquid moisture at $1241 \mathrm{~nm}$. Applications of NDWI include the analysis of the canopy stress, dense plant Leaf Area Index (LAI), plant productivity models, and fire sensitivity studies (Gao, 1996).

NDWI is defined by equation 1 :

$$
N D W I=\frac{\rho_{\text {Green }}-\rho_{N I R}}{\rho_{\text {Green }}+\rho_{N I R}}
$$

The NDWI value ranges from -1 to 1 . Generally, the green vegetation range is from -0.1 to 0.4 .

\subsubsection{Leaf Pigments}

Plant leaf contains three main groups of pigments including chlorophylls, carotenoids, and anthocyanins. These pigments are used in a variety of studies and are important for plant function and health. The concentration of plant pigments varies greatly according to the plant type. Plants with high concentrations of chlorophyll are generally very healthy. Chlorophyll is associated with greater light utilization and photosynthetic rates. Conversely, carotenoids and anthocyanin pigments may generally be seen in higher concentrations in less healthy plants due to stress and the onset of aging (Gitelson et al, 2002). Chlorophylls and carotenoids are the most important pigments being utilized in the process of plant photosynthesis.
Carotenoids have two well-known functions in photosynthesis: (1) as an auxiliary pigment in lightharvesting and (2) as a photoprotective agent against oxidative damage. One of the structural features of carotenoids is their ability to absorb visible light. The non-localized electrons are efficiently transferred to the energy chlorophyll (CHL) after undergoing a wilderness transformation in which a single state (s2) is produced, forming a singlet CHL with slightly higher energy (Delgado et al, 2000). In this study, two main pigments (chlorophylls and carotenoids) were taken into account to examine the vegetation pigments. NDVI (Fig. 2c) and CRI1 (Fig. 2b) indices were applied as indicatives of the status of chlorophylls and carotenoids.

\subsubsection{The Carotenoid Reflectance Index 1 (CRI1)}

The CRI1 index evaluates the spectral reflectance of plant leaves that is sensitive to carotenoids. Estimation of the leaves' carotenoid content is far more laborious than that of chlorophyll, because most leaves have a higher concentration of chlorophyll than carotenoids, with overlapping absorption peaks for chlorophyll and carotenoids. At $510 \mathrm{~nm}$, the mutual reflectance of carotenoids can be maximized, but this value is impacted by chlorophyll as well. The mutual reflectivity at $550 \mathrm{~nm}$ is used to eliminate the effect of chlorophyll. This is more purely influenced by chlorophyll alone. The higher the CRI1 value, the higher the concentration of carotenoids compared to chlorophyll.

Equation 2 is used to calculate CRI1:

$$
C R I 1=\frac{1}{\rho_{510}}-\frac{1}{\rho_{510}}
$$

The CRI1 index value can range from 0 to 15 or more. A typical range for green plants ranges from 1 to 12 .

\subsubsection{Normalized Difference Vegetation Index (NDVI)}

Among different vegetation indices, NDVI is surely one of the most recognized, and widely applied botanical indices. It is resistant to a variety of conditions by combining the establishment of normalized vegetation with the use of the best chlorophyll absorption and reflection areas. However, dense vegetation conditions can become saturated if LAI is high.

Equation 3 is used to calculate NDVI:

$$
N D V I=\frac{\rho_{N I R}-\rho_{\text {Red }}}{\rho_{N I R}+\rho_{\text {Red }}}
$$

NDVI values range from -1 to 1 , and healthy plants typically range from 0.20 to 0.80 .

\subsubsection{Dry or Senescent Carbon}

Plants contain various forms of carbon, including sugar, starch, cellulose, and lignin. The initial products of photosynthesis are sugars and starch. Plants generate 
cellulose and lignin transporting these carbon forms throughout their different parts. Cellulose is mainly used to create cell walls from plant tissue. Lignin is used in the most structurally strong plant parts such as leaf vacuoles, veins, woody tissues, and roots. The spectral properties of Cellulose and Lignin are manifested in the SWIR range of the light spectrum. Senescence signals the eventual stages of leaf development, kicking off the decomposition process essential for the leaf's nutrient recycling and redistribution. Plant growth regulators, germ cell division, and hormone levels all are internal factors that affect aging (Thomas and Stoddart 1980). In this study, the PSRI index (Figure 2c) was used to evaluate the dry or aging carbon status.

\subsubsection{Plant Senescence Reflectance Index (PSRI)}

PSRI was developed with the aim of increasing the index's sensitivity to the proportion of bulk carotenoids (such as alpha- and beta-carotene) to chlorophyll. The increased canopy tension (carotenoid pigment), canopy senescence, and plant fruit ripening are all indicators of the increased PSRI. The PSRI formula is shown in Eq. 4.

$$
\text { PSRI }=\frac{\rho_{\text {Red }}-\rho_{\text {Green }}}{\rho_{\text {NIR }}}
$$

The standard value range for the PSRI index is -1 to 1 , with its most common range of -0.1 to 0.2 for green vegetation coverages.

\subsubsection{Light Use Efficiency}

A Light use Efficiency Index is a metric that assesses how well vegetation utilizes incident light for photosynthesis (Gamon et al, 1997). The efficiency of light use is extremely associated with carbon absorption efficiency and plant growth rate. It is also linked to the partial absorption of photosynthetic active radiation (FAPAR). These VIs aid in the estimation of growth rates and output, which is beneficial in the implementation of precision agriculture policies. These VIs use visiblespectrum reflectance measurements to determine the vegetation's overall light use efficiency by taking advantage of relationships between various pigment forms (Gamon et al, 1997).

\subsubsection{Photochemical Reflectance Index (PRI)}

The Photochemical Reflectance Index (PRI), which is calculated using a narrowband spectroradiometer, is becoming increasingly often used as a photosynthetic efficiency measure. Variations in the number of carotenoid pigments from living leaves affect PRI (Peñuelas et al, 1992). Carotenoid pigments reflect the efficiency with which photosynthetic light is used, as well as the rate at which leaves absorb carbon dioxide per unit of energy consumed.

PRI is defined by Eq. 5:

$$
P R I=\frac{\rho_{531}-\rho_{570}}{\rho_{531}+\rho_{570}}
$$

The PRI index value ranges from - 1 to 1 from which a value range of -0.2 to 0.2 is typically ascribed to healthy vegetation.

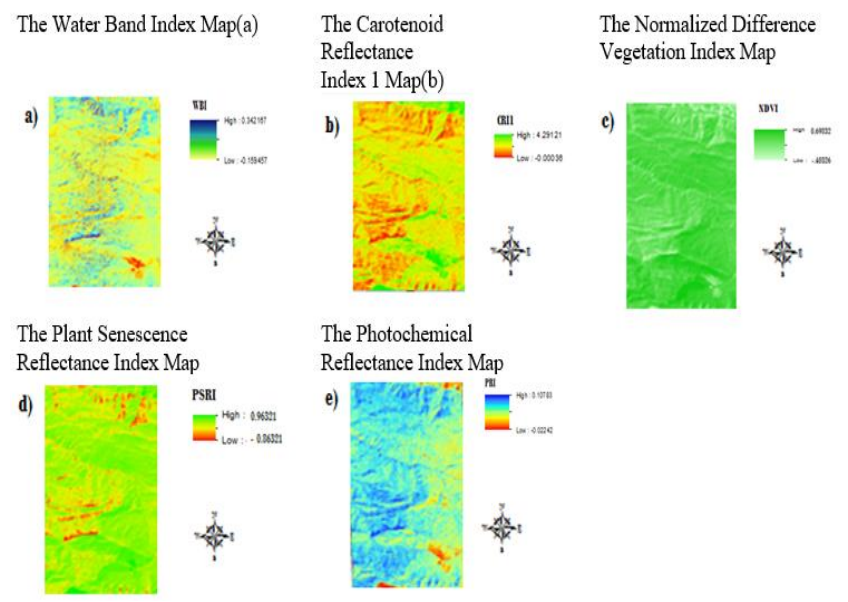

Figure 2. Study parameters' maps

\subsection{Fuzzy Degree Membership Functions}

A membership function (MF) is a curve that specifies how each point in the input space is converted to a membership value (or degree of membership) ranging from 0 to 1 . A fuzzy set is entirely characterized by its membership function.

Membership functions can be defined as a technique to solve fundamental problems based on experience rather than knowledge. In fuzzy theory, there is no optimal method to select the most suitable fuzzy membership functions and their respective parameters, so they are usually selected according to the preferences of decision-makers (Feizizadeh et al, 2013).

The Fuzzy Membership Tool converts the dataset into a range of values between 0 and 1 by using the membership probability of each input data based on their possibility of being a member of the given fuzzy set. While 0 is assigned to a position that is not a member of the specified set, 1 is assigned to a value that is a member of the specified set (Zadeh, 1968).

\subsection{Sigmoidal Membership Function}

The S-shaped membership function (also known as the "S" shape) generated using the cosine function may be the most widely utilized function in fuzzy set theory. In use, FUZZY requires a position of 4 points (along the $\mathrm{X}$ axis) that dominates the shape of the curve (Zadeh, 1968). The sigmoidal function was selected according to the preferences of the experts.

\subsection{Performing Scenarios}

\subsubsection{GIS}

Many functions have been introduced in Geographic Information Systems (GIS) to execute a variety of tasks such as evaluating available information, using it as a decision and support mechanism, and collecting and preserving the information as a whole. GIS may be utilized to create new field data from old data or to combine various aspects of a phenomenon to create a new understanding of it (Mesgari et al, 2008). 

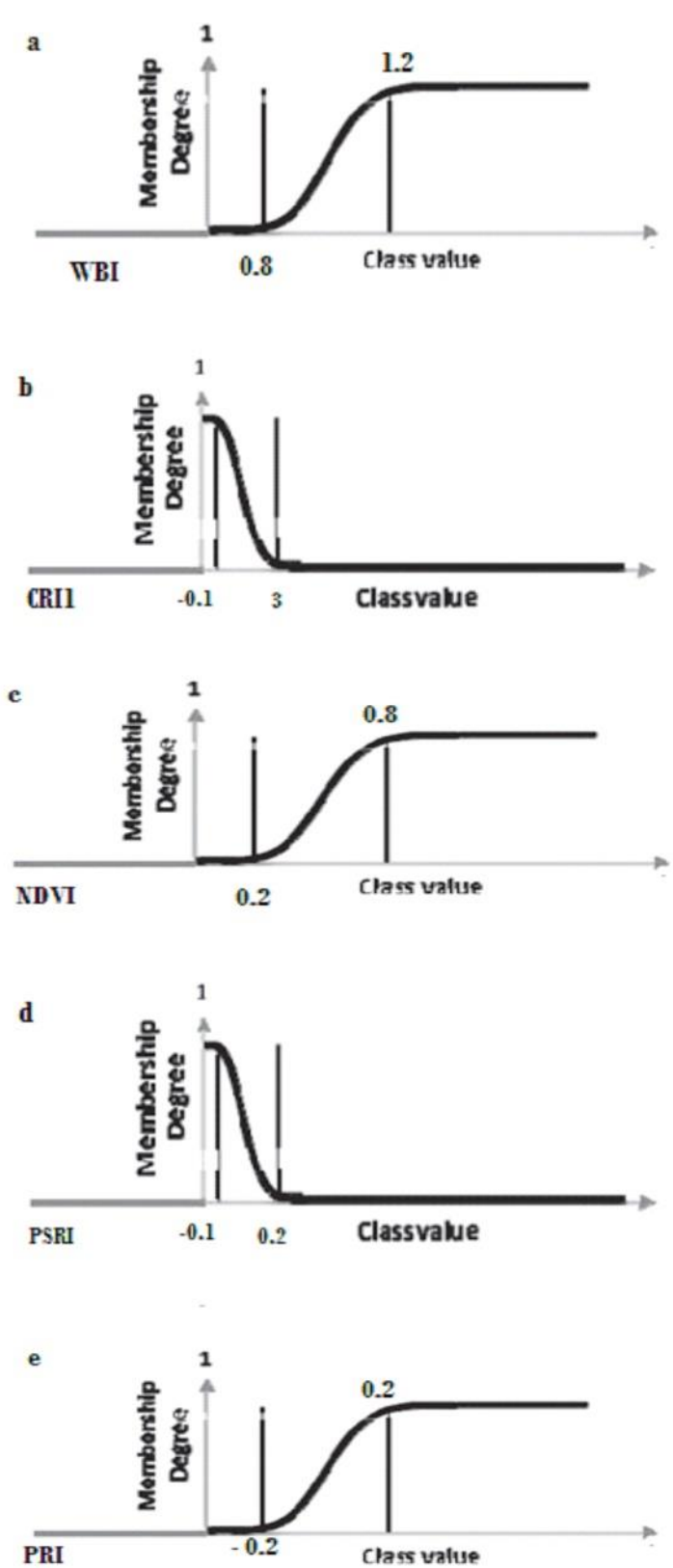

Figure 3. The Fuzzy based Sigmoidal membership graphs of the study parameters

GIS analysis can aid the portrayal of the spatial dispersion of observed forest conditions to find the causative parameters of the observed and categorized forest degradation process. Therefore, GIS, as a connecting link between environmental factors and effective management of forest, can serve as a tool for forest health assessment as well as forest managerial practices. As a planning tool, GIS can also play a predominant role in forest and land use monitoring studies in a broader sense (Jaiswal et al. 2001).

\subsubsection{Defining membership function}

As a fuzzy membership function, the widely used Sigmoidal function was implemented, and the overlay analysis was carried out based on the membership degree values allocated to each class of parameters using GIS coding (Figure 4).

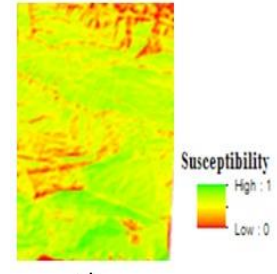

a)

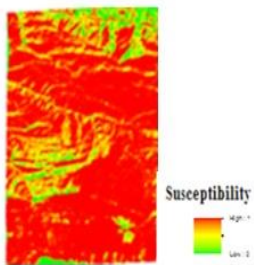

d) b)

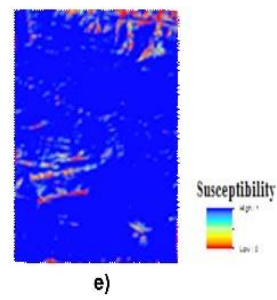

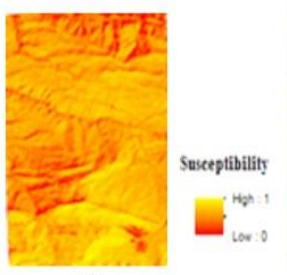

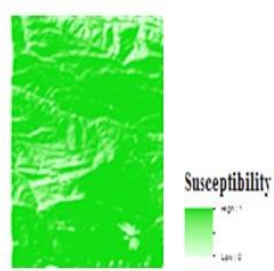

c)
Figure 4. Parameters Susceptibility Maps: WBI (a), CRI1 (b), NDVI (c), PSRI (d), and PRI (e).

\subsubsection{Fuzzy overlay}

The probability of a phenomenon pertaining to multiple groups of multi-criteria decision-making problems is examined using fuzzy overlay. It also examines the connections between the various sets' representatives (Baidya et al., 2014; Akgun et al. 2012).

The traditional applications of overlay analysis such as site selection and land suitability modeling can take the benefits of the GIS-based fuzzy logic as a technique. In a multi-criteria overlay analysis, the Fuzzy Overlay technique may be used to examine the probability of a phenomenon belonging to different sets. Fuzzy Overlay now not only pinpoints which fuzzy set the phenomenon can belong to, but it also analyzes the relationships between the contributors to the various sets. The methods for combining data based on fuzzy theory analysis are listed under the overlay form. Each approach helps you to look at which input parameters each cell belongs to. Fuzzy And, Fuzzy Or, Fuzzy Product, Fuzzy Sum, and Fuzzy Gamma are some of the available methods. Each method focuses on a particular feature of each cell's membership in the various input parameters.

\subsubsection{Fuzzy And}

The Fuzzy And overlay return the data set's minimum value for the cell position. This method is useful when determining the least common denominator for all input parameters membership. In its estimation, Fuzzy And employs the following function:

Fuzzy And Value $=\min (\arg 1, \ldots, \operatorname{argn})$

\subsubsection{Fuzzy Or}

The whole value range of the fuzzy sets is returned by the Fuzzy Or function. This method is useful for determining the greatest membership range for all of the input parameters. In the assessment, Fuzzy Or employs the following function:

Fuzzy Or Value $=\max (\arg 1, \ldots, \operatorname{argn})$ 


\subsubsection{Fuzzy Product}

The Fuzzy Product function multiplies each value of the fuzzy set for all of the input parameters. The final product is smaller than all of the inputs, and the sum can be very minimal when a member of many sets is used. In the evaluation of Fuzzy Product, the following function is used:

Fuzzy Product Value $=\operatorname{product}(\arg 1, \ldots, \operatorname{argn})$

\subsubsection{Fuzzy Sum}

The Fuzzy Sum overlay combines the fuzzy values of all the sets of which the cell position corresponds. The resulting sum is an increasing linear combination equation based on the total number of parameters used in the analysis. In its estimation, Fuzzy Sum employs the following function:

Fuzzy Sum Value $=1-\operatorname{product}(1-\arg 1, \ldots, 1-\operatorname{argn})$

\subsubsection{Fuzzy Gamma}

The mathematical product of the Fuzzy Product and the Fuzzy Sum functions defines the Gamma overlay function where each of the Product and Sum functions is lifted to the power of gamma. As an example, consider the following generalized function:

$\mu(\mathrm{x})=($ Fuzzy Sum $) \gamma *($ Fuzzy Product $) 1-\gamma$

The Fuzzy AND overlay feature was used in this analysis to pick places that had at least a 0.5 or greater suitability for all of the parameters.

\section{RESULTS and DISCUSSION}

The fuzzy membership boundaries were defined based on the recommendations of four experts. The forest health map (Fig 5) shows that the research area's southern and western portions have a "very low" to "low" forest health index, while the remainder has a "medium" to "very high" health index.

The findings of this study shows that Ramsar forest health status is highly dynamic and bears a spatial nature. The findings also show that improving our knowledge of the causes that impact forest health is critical for forest management and prioritizing forest health-related interventions.

\section{CONCLUSION}

In this study, Ramsar forest health was quantified in terms of canopy water component, carotenoid and chlorophyll pigments in plant foliage, plant senescence, and photochemical reflectance. A GIS-based fuzzy analytical hierarchy process (AHP) was used to solve the "fuzziness" of the spatial dataset and also to integrate the subjective judgments in the modeling process. Results are quite useful in delineating potential "forest health" zones at a district level. The findings of the current study signal the applicability of the used methodology for strategic planning of forest health. It may also be applied to assess the health susceptibility of any vegetation coverage.
Overall, the findings of this study demonstrate the potential of GIS technology, MCDM, and its viability for the assessment of forest health by integrating objective as well as subjective data in a Fuzzy Logic.

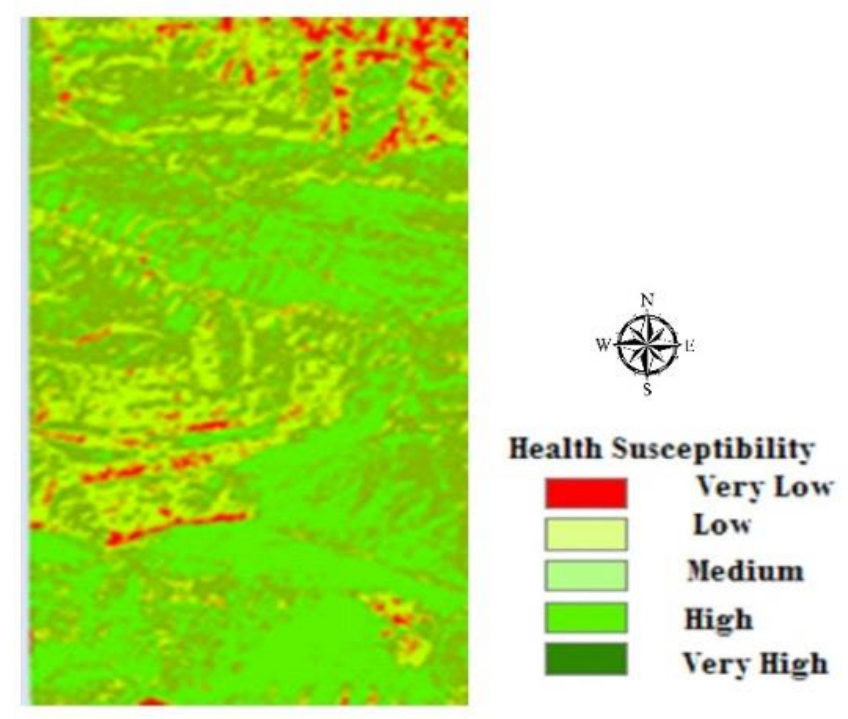

Figure 5. Forest Health Susceptibility Map

\section{Author contributions}

Khalil Valizadeh Kamran: Conceptualization, methodology, analysis, Behnam Khorrami Mehtodology, analysis, writing and review

\section{Conflicts of interest}

The authors declare no conflicts of interest.

\section{REFERENCE}

Akgun A, Sezer E A, Nefeslioglu H A, Gokceoglu C \& Pradhan B (2012). An easy-to-use MATLAB program (MamLand) for the assessment of landslide susceptibility using a Mamdani fuzzy algorithm. Comput Geosci, 38, 23-34.

Baidya P, Chutia D, Sudhakar S, Goswami C, Goswami J, Saikhom V, ... \& Sarma K K (2014). Effectiveness of Fuzzy Overlay Function for Multi-Criteria Spatial Modeling-A Case Study on Preparation of Land Resources Map for Mawsynram Block of East Khasi Hills District of Meghalaya, India. Journal of Geographic Information System, 6, 605-612.

Bolle H J, Eckardt M, Koslowsky D, Maselli F, Miralles J M, Menenti M, ... \& Van de Griend A A (Eds.). (2006). Mediterranean landsurface processes assessed from space. Berlin, Heidelberg: Springer Berlin Heidelberg.

Boyer J S (1982). Plant productivity and environment. Science, 218(4571), 443-448.

Carter G A \& Miller R L (1994). Early detection of plant stress by digital imaging within narrow stresssensitive wavebands. Remote Sensing of Environment, 50(3), 295-302.

Ceccato P, Flasse S, Tarantola S, Jacquemond S \& Gregoire J M (2001). Detecting vegetation water content using reflectance in the optical domain. Remote Sensing of Environment, 77, 22-33. 
Chapin F S (1991). Integrated responses of plants to stress. BioScience, 41, 29-36.

Colombo S J \& Parker W C (1999). Does Canadian forestry need. Physiology research For. Chronicle, 75, 667673.

Cox E (2005). Fuzzy modeling and genetic algorithms for data mining and exploration. Elsevier.

Delgado-Vargas F, Jiménez A R \& Paredes-López O (2000). Natural pigments: carotenoids, anthocyanins, and betalains-characteristics, biosynthesis, processing, and stability. Critical reviews in food science and nutrition, 40(3), 173-289.

Feizizadeh B, Blaschke T \& Roodposhti M S (2013). Integrating GIS-based fuzzy set theory in MultiCriteria evaluation methods for Landslide susceptibility mapping. International Journal of Geoinformatics, 9(3).

Feizizadeh B, Blaschke T \& Zamani H (2012). GIS-based ordered weighted averaging and Dempstershafermwthod for landslide susceptibility map in Urmia lake basin, Iran. International journal of digital earth. DOI: 10:1080. 17538,2012.

Feizizadeh B, Blaschke T (2012). Comparing GIS Multicriteria decision analysis for landslide susceptibility mapping for the Urmia lake basin Iran, (IGARSS)2012 IEEE International 10.1109, IGARSS 2012.

Gamon J A \& Surfus J S (1999). Assessing leaf pigment content and activity with a reflectometer.New Phytol1999;143:105-117.

Gamon J, Serrano L \& Surfus J S (1997). The photochemical reflectance index: an optical indicator of photosynthetic radiation use efficiency across species, functional types, and nutrient levels. Oecologia, 112(4), 492-501.

Gao B-C (1996). NDWI - A normalized difference water index for remote sensing of vegetation liquid water from space. Remote Sensing of Environment, 58, 257266.

Gitelson A A, Zur Y, Chivkunova O B \& Merzlyak M N (2002). Assessing carotenoid content in plant leaves with reflectance spectroscopy. Photochemistry and photobiology, 75(3), 272-281.

Iliadis L (2007). Intelligent Information Systems and Applications in Risk Estimation (in Greek) Stamoulis Publishing Thessaloniki, Greece

Jackson R D (1986). Remote sensing of biotic and abiotic plant stress. Annual review of Phytopathology, 24(1), 265-287.

Jaiswal R K, Mukherjee S, Raju D K (2001). Forest fire risk zone mapping from satellite imagery and GIS. International Journal of Applied Earth Observation and Geoinformation, 4, 1-10.

Keeney R L, Raiffa H \& Meyer R F (1993). Decisions with multiple objectives: preferences and value trade-offs. Cambridge university press.

Leondes C T (1998). Fuzzy logic and expert systems applications. Elsevier.
Malczewski J (2002). Fuzzy screening for land suitability analysis. Geographical and Environmental Modelling 6, 27-39. DOI: 10.1080/13615930220127279.

Mesgari M S, Pirmoradi A \& Fallahi G R (2008). Implementation of overlay function based on fuzzy logic in spatial decision support system. World Applied Sciences Journal, 3(1), 60-65.

Moroni M, Lupo E, Marra E \& Cenedese A (2013). Hyperspectral image analysis in environmental monitoring: setup of a new tunable filter platform. Procedia Environmental Sciences, 19, 885-894.

Peñuelas J, Savé R, Marfà 0 \& Serrano L (1992). Remotely measured canopy temperature of greenhouse strawberries as indicator of water status and yield under mild and very mild water stress conditions. Agricultural and Forest Meteorology, 58(1-2), 63-77.

Pomerol J C \& Barba-Romero S (2000). Multi criterion decision in management: Principles and management. Boston: Kluwer Academic

Saaty (1968). Fuzzy sets. Information and Content,8, 338-356. DOI: 10.1016/S0019-9958(65)90241-X.

Sadiq R \& Husain T (2005). A fuzzy-based methodology for aggregative environmental risk assessment: A case study of drilling waste. Environmental Modelling\&Software, 20, 33-46. DOI: 10.1016/j.envsoft.2003.12.007.

Sampson P H, Mohammed G H, Zarco-Tejada P J, Miller J $\mathrm{R}$, Noland T L, Irving D, Treitz P M, Colombo S J \& Freementle J (2000). The bioindicators of forest condition project: Aphysiological, remote sensing approach. For. Chronicle, 76(6), 941-952.

Sims A D \& Gamon J A (2002). Relationships between leaf pigment content and spectral reflectance across a wide range of species, leaf structures and developmental stages. Remote Sensing of Environment, 81, 337- 354.

Thenkabail P S, Smith R B \& De Pauw E (2002). Evaluation of Narrowband and Broadband Vegetation Indices for Determining Optimal Hyperspectral Wavebands for Agricultural Crop Characterization. Photogrammetric Engineering and Remote Sensing, 68(6), 607-621.

Thomas H \& Stoddart J L (1980). Leaf senescence. Annual review of plant physiology, 31(1), 83-111.

Verstraeten W W, Veroustraete F \& Feyen J (2006). On temperature and water limitation of net ecosystem productivity: Implementation in the C-Fix model. Ecological Modelling, 199(1), 4-22.

Yan D \& Yang X (2011). Health assessment of forest ecosystem based fuzzy synthesis assessment. In 2011 International Conference on Electric Technology and Civil Engineering (ICETCE) (4941-4944). IEEE.

Zadeh L A (1968). Fuzzy sets. Information and Content,8, 338-356. doi: 10.1016/S0019-9958(65)90241-X.

Zarco-Tejada P J, Miller J R, Mohammed G H, Noland T L \& Sampson P H (2002). Vegetation Stress Detection through Chlorophyll a $+\mathrm{b}$ Estimation and Fluorescence Effects on Hyperspectral Imagery, Journal of. Environ. Qual, 31, 1433-1441 